\title{
Integrating Collaborative Concept Mapping Tools with Group Memory and Retrieval Functions
}

\author{
H. Ulrich Hoppe \& Katrin Gaßner \\ University of Duisburg \\ \{hoppe,gassner\}@informatik.uni-duisburg.de
}

\begin{abstract}
The provision of shared visual representations is considered to be an important facilitator for creative processes in group working and learning scenarios. Although reusability is an inherent feature of computerised representations in general, a comparative analysis of existing tools shows clear deficits in this respect. We are convinced that reusability and thus sustainability of the results of co-constructive group work can be much enhanced by integrating visual environments with functions for indexing, archiving and retrieval to support the construction of group memories. This is exemplified with a new tool which supports creative working groups in the area of "trend monitoring" and technology transfer. This is an example of organisational learning, but also more standard learning scenarios may benefit from this technology.
\end{abstract}

\section{Keywords}

collaborative visual languages, co-construction, group memory, life-long learning

\section{INTRODUCTION}

The co-constructive use of shared visual representations is considered to be an important facilitator for creative processes in group working and learning scenarios. The perspectives range from small learning groups in educational scenarios to learning organisations. The basic function supported by shared visual representations is externalisation. According to Nonaka (1994), externalisation plays an important role in "organisational knowledge creation", namely in that it supports the transition from tacit, individual to explicit knowledge.

In computer-supported collaborative learning scenarios, shared workspaces with more or less specific visual representations are used to facilitate and to enrich synchronous communication and collaboration. Typical applications are group discussions (Conklin \& Begeman, 1987; Streitz et al., 1998; Hoppe et al., 2000; Gaßner, 2001), scientific argumentation (Suthers et al., 1997), scientific modelling (v. Joolingen, 2000; Pinkwart et al., 2001), group design (Stahl, 2000). According to Hoppe \& Plötzner (1999) shared workspace environments support the following types of cognitive processes within the learning group:

- coordination of individual contributions or action through external constraints of the shared workspace,

- reification of contributions as manipulable objects,

- "mise en relation" (in a Piagetian sense) by visually relating individual contributions to each other using a spatial metaphor,

- reuse of group results (e.g., for reflection, comparison, further elaboration).

Reusability is, of course, an inherent feature of computerised representations in general. Yet, a critical analysis of existing tools shows that it is usually not very explicitly supported (see below). The main concern appears to be the provision of a rich, flexible and expressive environment for the collaborative sessions, i.e., the collaborative session is essentially conceived as a closed event. We are convinced that reusability and thus sustainability of the results of co-constructive group work can be much enhanced by integrating visual environments with functions for indexing, archiving and retrieval to support the construction of group memories.

Similar orientations have been developed from a knowledge management perspective (e.g., Borghoff \& Pareschi, 1998) or from the needs of supporting collaborative design activities (Stahl, 2000). A combination of concept mapping techniques and discussion support in the IBIS tradition with group memory functions has also been pursued in the Compendium approach (Selvin et al., 2001). Although we share the basic orientations, our approach is different in so far as it evolved as a smaller bottom-up activity, driven by the practical need of adapting an existing collaborative visual language framework to the needs of certain creative group processes. 


\section{ANALYSIS OF EXISTING SYSTEMS AND TOOLS}

The following synopsis and comparison is targeted at collaborative visual language environments. In general, we see these as characterised by the following features:

- provision of shareable representations in visual, graph-structured format

- co-constructive editing facilities

- $\quad$ support of certain methodologies for brainstorming, knowledge management, learning, etc.

An early account of the notion of "visual languages for co-operation" which is very much congruent with our understanding has been given by Lakin (1990).

\section{Examples}

The gIBIS system (Conklin \& Begemann, 1987) is an early example of using visual languages to represent and elaborate arguments during a design process. Both are used cooperatively but asynchronously. At that state they did not use any operational semantics but took advantage from using an intended structure. The visualisation of the arguments and ideas is expected to make the design process more rational. As a spin-off, a documentation is obtained and important concepts might be recognised.

Another example that stresses more the aspect of workflow semantics is the SEPIA system (Streitz et al., 1992). SEPIA, developed for the cooperative design of hypermedia documents, offers four types of visual languages: planing, argumentation, content and a rhetorical space. Particularly the argumentation space uses a graph representation derived from S. Toulmin's argument patterns. The generation of some types of contributions in one workspace causes the automatic generation of an adequate object in another, which is an invitation for further exploration. The SEPIA system has later been modified to support face-to-face meetings and discussions. This system called Dolphin supports also free-hand-drawing and handwriting (Streitz et al., 1998). Since Dolphin is based on SEPIA, though not every SEPIA feature is offered, we will treat the "union" of the two systems as one.

In the CSILE environment (Scardamalia et al., 1992) learners construct knowledge cooperatively by creating a base of learning material which integrates graphics and text. This "community database" is extended through critical annotations of documents which capture a flow a discussion and knowledge refinement. The working procedure is distributed and asynchronous. Documents in the database are not linked but can be searched which allows using existing material in new contexts. Although CSILE is not primarily based on a structured visual representation, we have included it in this comparison since it a collaborative learning environments with interesting group memory features.

The Belvedere system (Suthers et al., 1995) was designed to teach students scientific argumentation. It uses a graph notation similar to SEPIA's rhetorical space. It offers two types of content objects, data and hypotheses. By using the system, argumentation rules such as "hypotheses without supporting data are not accepted" should be understood. Via an agent, the system analyses the developed structure and points out missing relations. In recent versions, co-operation is supported by the means of a shared workspace environment (Suthers et al., 1997).

The CardBoard environment (Gaßner et al., 1998) allows for creating multiple visual languages by parameterising a general shared workspace environment. A particular language profile specifies the syntax of the respective visual language in terms of a set of relations ("connector cards"), their argument slots, and the basic object types ("content cards"). To add semantics in terms of domain models or knowledge bases, an interface is provided that transfers actions from the visual language environment to the semantic plug-in component (Mühlenbrock, Tewissen \& Hoppe, 1997). This architecture allows for flexibly defining semantically enriched tools, such as, e.g., a cooperative editor and simulator for Petri Nets (Wagler, 1998) or a discussion environment (Gaßner, 2001). The same plug-in architecture has also been used for analysing action patterns in collaborative learning and problem solving scenarios (Mühlenbrock \& Hoppe, 1999).

\section{Comparison}

We will use the following criteria to classify collaboration support systems under the perspective of supporting knowledge management and a knowledge flow, e.g. for organisational learning (criteria are not meant to be descriptive not to judge the systems as better or worse):

1. Domain independence: Typically special methods such as cooperative text planning, brainstorming, arguing, etc., need special tools. The question is how flexibly the system can be adapted to different representations and forms of usage. 
2. Shared workspaces (and shared objects) are essential for cooperative work in distributed, both distance and face-to-face, scenarios. The reification feature depends on shared objects.

3. Applicability in synchronous mode.

4. Applicability in asynchronous mode (e.g., discussion threads or group archives).

5. Flexible definition of language syntax: It should be easy to define different visual representations on a syntactic level without having to reprogram the system.

6. Externally definable semantics: The provision of mechanisms to plug-in components defining object semantics or operational semantics (e.g. in modelling applications). Enables intelligent background processes to support the workflow processes as well constraint-checking of solutions.

7. System driven interpretations of states or processes can support special perspectives on the visual maps. This can increase the awareness for the users.

8. Repositories can be provided not only for storing and accessing intermediate or final products, but also to store processes or action histories. Can be combined with annotation facilities.

9. Replicated architectures in which application data are synchronised but maintained autonomously in several places of the distributed environment allow for flexible sharing models (co-existence of private and public workspaces) and robustness (recovery).

10. Integration of common media formats (text, graphics, sound, animations) is important for a general knowledge management because not each information occurs in one special format used for a specialised tool.

11. Free-hand input: In our experience, free-hand input facilities (hand writing, sketching) are particularly wellsuited to support informal, creative processes, as e.g. in brainstorming sessions were typing is disruptive.

12. Explicit support of process models means that the system of different working phases or working goals are supported, usually in accordance with certain discussion styles or learning methods.

13. Media repositories: Independent of the products developed with the specific tool, repositories of foreign material in common formats can be provided. Here, good retrieval functions are of particular interest.

Table 1: Comparison of collaborative environments

\begin{tabular}{|l|c|c|c|c|c|}
\hline & Belvedere & CardBoard & CSILE & $\begin{array}{c}\text { SEPIA/ } \\
\text { Dolphin }\end{array}$ & gIBIS \\
\hline Domain independence & o & + & + & + & + \\
\hline Shared workspaces & + & + & - & + & - \\
\hline Asynchronous work & $?$ & - & + & + & + \\
\hline Synchronous communication & + & + & - & + & - \\
\hline $\begin{array}{l}\text { Flexible definition of } \\
\text { language syntax }\end{array}$ & - & + & - & - & - \\
\hline Externally definable semantics & - & + & - & - & - \\
\hline $\begin{array}{l}\text { System driven interpretation of } \\
\text { states or processes (filters) }\end{array}$ & $\begin{array}{c}\text { through } \\
\text { agents }\end{array}$ & $\begin{array}{c}\text { through } \\
\text { plug-in }\end{array}$ & - & - & + \\
\hline Repositories & $\begin{array}{c}\text { product } \\
\text { repository }\end{array}$ & $\begin{array}{c}\text { action protocol, } \\
\text { no product DB }\end{array}$ & + & $\begin{array}{c}\text { product } \\
\text { repository }\end{array}$ & - \\
\hline Replicated data maintenance & - & + & - & - & - \\
\hline $\begin{array}{l}\text { Process models } \\
\text { (workflow support) }\end{array}$ & $\begin{array}{c}\text { argumentation } \\
\text { patterns }\end{array}$ & - & (implicit) & $\begin{array}{c}\text { different visual } \\
\text { languages } \\
\text { for diff. phases }\end{array}$ & + \\
\hline $\begin{array}{l}\text { Integration of common } \\
\text { media formats }\end{array}$ & - & + & + & + & - \\
\hline Free hand input & - & + & - & + & + \\
\hline Media repositories & $\begin{array}{c}\text { (in learning } \\
\text { material) }\end{array}$ & - & + & - & - \\
\hline
\end{tabular}


The distribution of positive marks indicates that the design of these systems was very much targeted at the first group of features. The second group of criteria is inspired by the engineering of standardising and "factoring out" certain generic functions that has guided an essential portion of our work on CardBoard. Replication (criterion 9) is of more general interest since it allows for very flexible forms of interaction in synchronous mode.

Generally, the last two groups of criteria reflect in some way or other system features which are desirable to support the sustainability and reusability of the work done in the collaborative environment. Yet looking at the distribution of marks, here, gives a too positive picture. Even if specific support is provided, there is usually one restriction: information access functions and "indexed archives" are usually confined to the products of the system itself, i.e., there is no real interoperability to share knowledge elements with external sources (e.g., by importing resources from the web or by exchanging elements with a general corporate memory). So, the main problem is that current systems are not open! We will particularly address this challenge in the case study reported below.

\section{Extensions of collaborative visual language environments}

In most cases, the development of more or less generic visual language environments aims at obtaining easy-tocommunicate visualisations rather than at providing (seem-)formal machine-interpretable representations. The focus is on interactive tools and interfaces to represent a domain or support a certain task, not on system-internal structure and semantics. In contrast to the generic cooperative environments mentioned, specific tools like STELLA (http://www.hps-inc.com) or Rational Rose (http://www.rational.com) provide visual interfaces for existing model semantics, as e.g. "system dynamics" in the case of STELLA or UML in the case of Rational Rose. They (implicitly) use visual languages and provide a complete semantic interpretation to augment relations between nodes by operations.

A recent example of a collaborative learning environment based on a domain-specific visual language is the COLER system (Constantine-Gonzalez \& Suthers, 2000). COLER supports the co-construction of entityrelationship (ER) models for database modelling. A current redesign and reimplementation of the CardBoard environment (JavaCardBoard) is focussed at supporting different kinds more or less formalised visual languages, including Petri nets and system dynamics models, which can be mixed and combined with free-hand annotations (Pinkwart et al., 2001).

It was also Suthers (1999) who studied and reflected the impact of different visual tools and representations on collaborative learning discourse ("representational bias"). Typically, concrete representation systems come with inherent characteristics which favour or inhibit certain aspects and thus influence learning and working styles.

\section{CASE STUDY: SUPPORTING TREND MONITORING IN SMALL TEAMS}

In the following we will describe a modification of the CardBoard environment which has been developed to support small working groups monitoring new trends in science and technology. As a result, a new system, called FreeStyler has been developed based on the requirements defined and assessed with two application sites: the technology transfer department (TTD) of our university and a "pilot development group" in a large European company. Although the scenario is not primarily a learning scenario, it involves aspects of organisational learning and the incremental building of a group memory.

Since the role of TTD is to act as a transmission interface between university and industry, a "trend" cannot be determined by retrospective of cases but rather as a combination of existing information and new requests the TTD receives. Information connections result in a structure that is the TTD-perspective on a trend and its information context. The daily work of TTD consists largely of personal consulting through which individual solutions for cooperation opportunities have to be found (Figure 1). More or less static information prepared for the web turned out to be not adequate for such a dynamic process and was also not well-suited to support internal information exchange. The consulting process includes phases in which only hand-written notes are useful, phases where information is exchanged and phases in which multiple media have to be put into a common context.

From a representational and media point of view, the system supports free-hand input to be able to smoothly support informal creative processes, structured representations (discussion graphs, models) as well as certain visual representations of data collections. All these features are equally interesting for many learning scenarios such as scientific argumentation and modelling. 


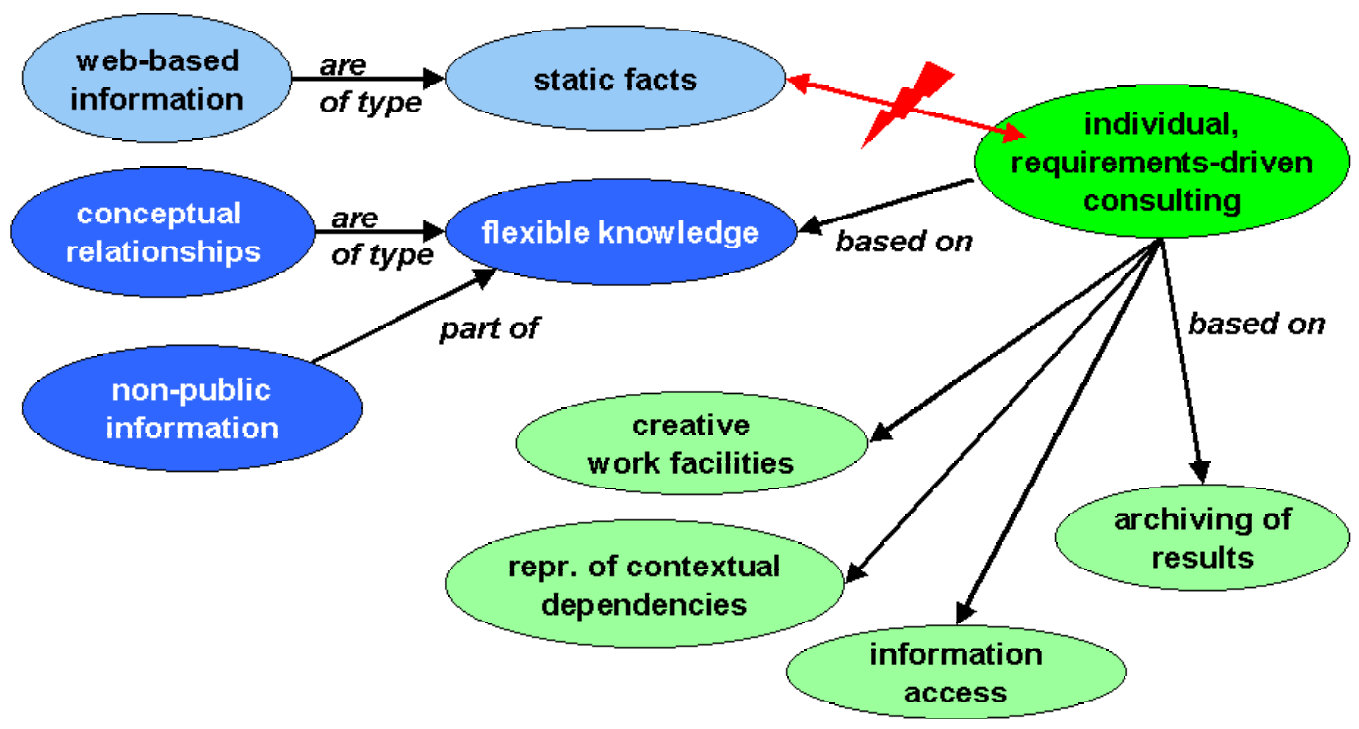

Figure 1: Basic dependencies and requirements

For the TTD, trend monitoring and consulting is a continuous, long term process as shown in Figure 2. During a first contact, in the majority of cases by the means of a telephone, hand-written notes are put that are the basis for the next meetings. Open questions are fixed to be further annotated and elaborated on, new documents are added or old documents are linked. Such a preparation of materials represents the input for a later meeting that might lead to another accumulation of media and materials.

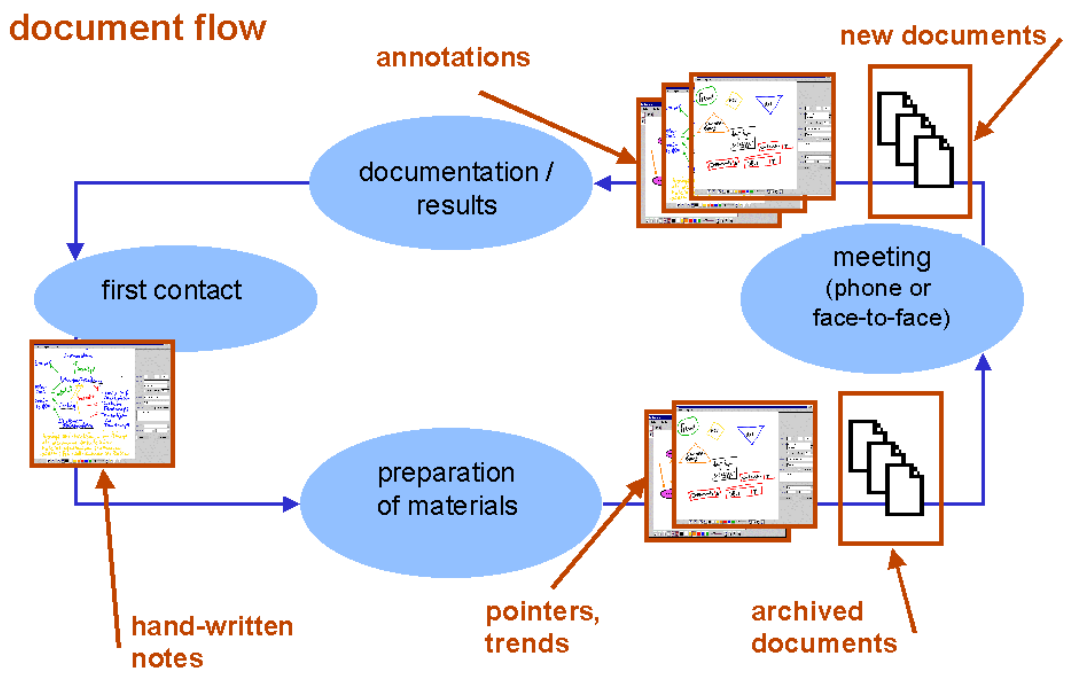

Figure 2: Document flow related to trend monitoring

It is a general observation, that externalisation forms as the bottleneck of knowledge management projects that try to integrate dynamic information. In our approach, we try to provide seem-structured, heterogeneous representations which can be refined and grow. The document flow does not introduce new procedures or formalities as compared to the original process which was only partially computerised. The benefit that we expect to gain by providing integrated support for this process lies in the avoidance of representational discontinuities and interoperability deficits. 


\section{Technical features}

In the following, the main features of the TTD knowledge management system are introduced. Figure 3 shows the overall system architecture and main interactions. The user interface is introduced in the next subsection which also includes an example of the evolution of a document.

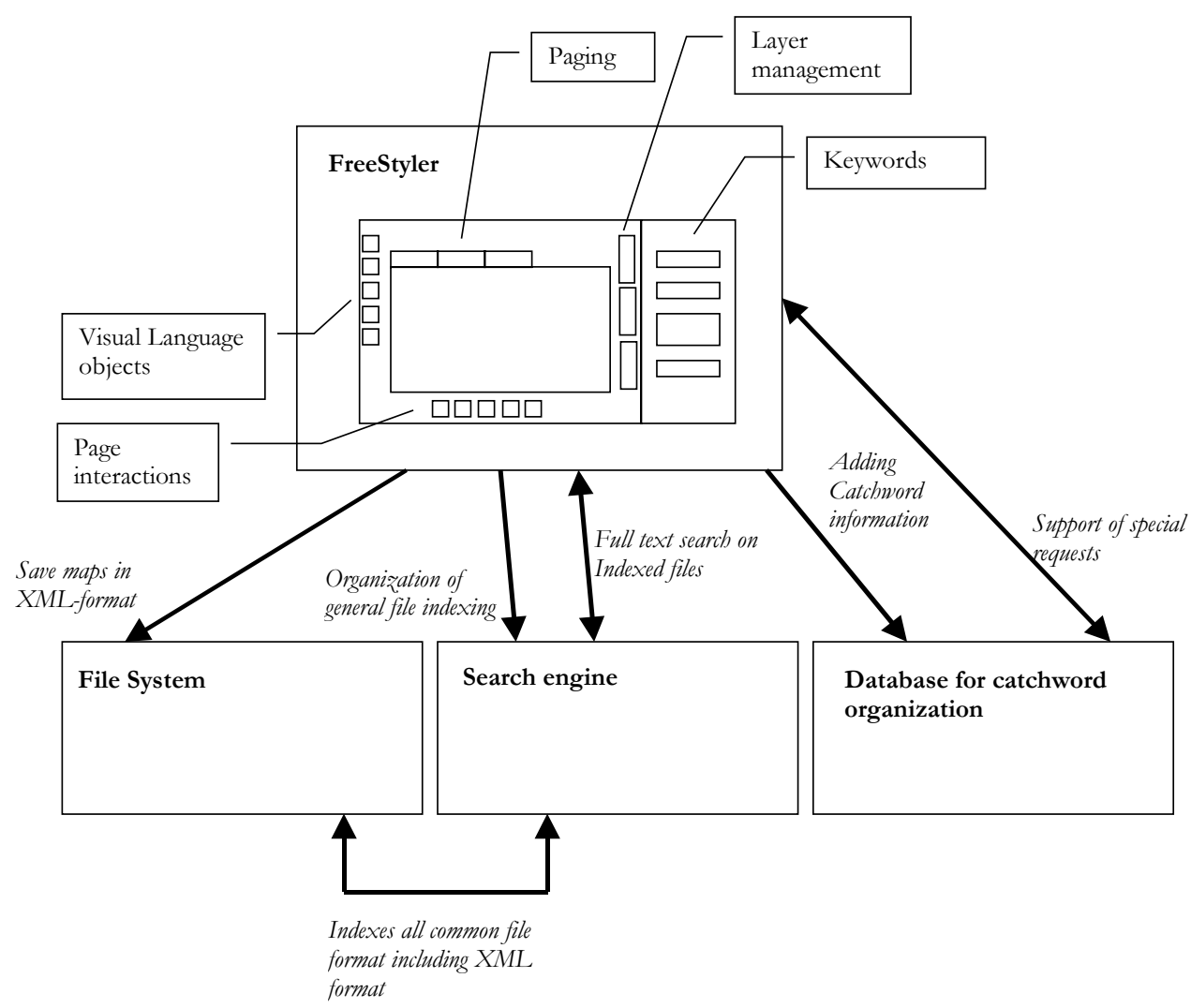

Figure 3: System overview

\section{Visual language}

FreeStyler is a Java application that interacts with a powerful search engine (Verity, cf. www.verity.com) and a database system. A visual language is used to structure the content. Predefined shapes provided on a palette represent different input types to distinguish different elements in the elaboration and analysis of an issue. A clear distinction of contribution types using shapes and colours supports the interpretation of material by other users. Hand-written annotations can be added flexibly.

\section{Easy paging through workspace elements}

The application offers pages to structure a document. By the use of "page tabs" (on top of the workspace) it is easy to go directly to a page without scrolling. Pages can be named by the user. Each page offers again a couple of layers in order to stratify different levels of input. There are layers for hand-written input and for the entries. The layer sequence can be changed and moreover, they can be off. This allows for representing different levels of detail and different perspectives.

\section{User-definable hyperlinks}

User-definable internal links can be added as specific elements to the workspace. Their content is the link address which points to another page represented by its marker. It is possible to add multiple markers to pages which are shown as headers on the page tabs. Another entry type can also be used for external links to URLs and files. According to the file type, an appropriate viewer is selected and invoked when the node is activated.

\section{Embedding of objects representing data collections or documents}

The same type of entries that are used for external links are also used to represent document collections. 


\section{Embedding query objects}

List entries also contain query results of the search engine which realises a full text search on the common file formats e.g. .doc, .pdf, .xml, .txt, etc. Using the Java Native Interface the query is handled by a Dynamic Link Library which uses the API of the search engine. The result is given back to the application and the retrieved documents are listed in the list entry. There they can be accessed interactively. The associated database offers other query possibilities: It allows for associating keywords with files and to ask for newest keywords and e.g. connections among keywords. To express these queries, the Java-SQL interface is used.

\section{XML format}

The developed materials are called "maps", following the idea of concept mapping. They are saved to XML format using the Java DOM interface. In connection with the search engine which is also able to search XML format, the maps can be searched as well. Using the keywords, which are also saved to the XML files, maps can be interrelated using the search engine.

The TTD working process includes both synchronous and asynchronous co-operation. The search mechanisms support also the asynchronous access to documents. The development of materials by different authors is intended and supported. Integrated mail facilities allow for notifying group members about new occurrences. To reach more flexibility, documents can either be referred inside the mail or the whole document can be send. For the latter, both is possible either to send the XML file or a version that is transformed to graphic format. Mail lists can be defined during the work with a document for automatic notification.

Currently, FreeStyler is either used individually or in face-to-face meetings with a big interactive display. Mostly, co-operation occurs in asynchronous mode.

\section{Example}

The following group of figures show the process of developing a map. In Figure 4, FreeStyler contains first notes that have been entered during a telephone call. A person from industry has asked for a contact to a university group that works on the topic of fuel cells. The notes include typical questions of the TTD at that stage for more detailed elaboration. The "client" asked for references to former projects and for the topic in general. He is also interested in published results. The right hand side of the application window contains the keyword entry section (for indexing). Here, also name and address of the contact person are noted.

Figure 5 shows the result of a first preparation phase wherein a user adds some information requests. Subsequently, some pages have been added. The links on the page (small circles) relate the notes of the call to this new material put to the linked pages.

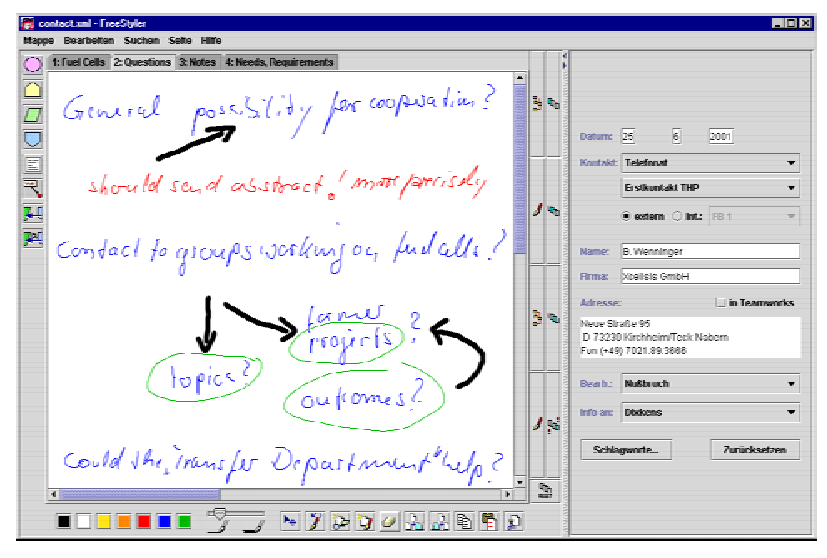

Figure 4

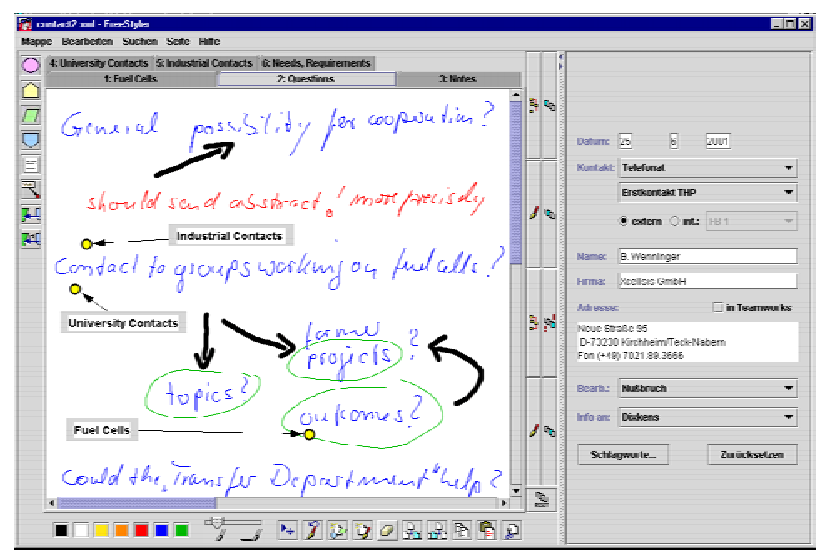

Figure 5

Figure 6 represents a page with institutional contacts. The institutions are listed in entries that are again related to corresponding URLs. Later, during a meeting, it is a very easy to follow that link for more information and, e.g., to discuss the relevance of such a contact. This is a typical activity during a meeting with experts because the TTD itself does not have detailed content expertise for each scientific field or topic and cannot decide on beforehand which contact might be interesting for an industry partner. On the right hand side of the screen, a web browser has 
been invoked with a requested URL. If the content of the list node had been a file path, the appropriate viewer would have been called to show the file by double clicking on the node.

Figure 7 shows a more content oriented page that might be a central part of a trend documentation. The map represents information on "fuel cells". Therefore, general concepts and background information are combined in a structured way. In this figure, multiple entries are integrated: Some entries represent information associated with the trend. Another is a graphical entry. The opened dialogue allows for searching the indexed documents of the file system concerning the requested concept. This dialogue covers a list node with results from a previous different request.

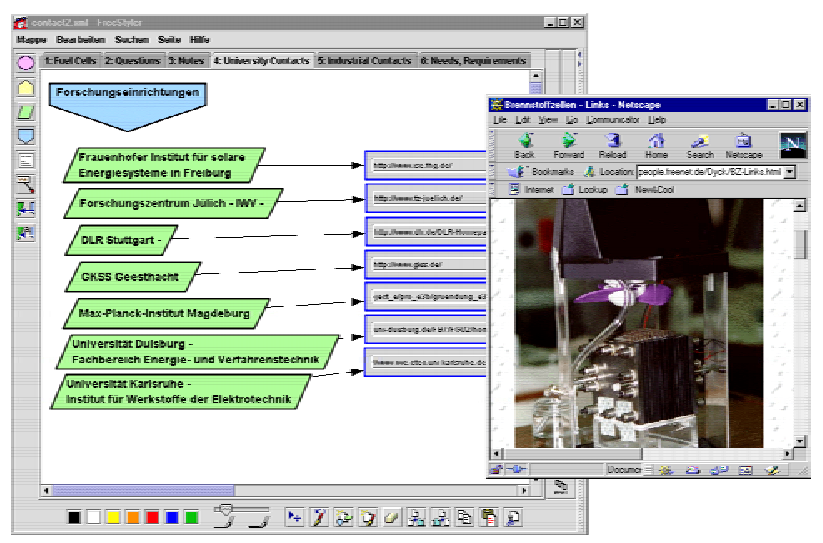

Figure 6

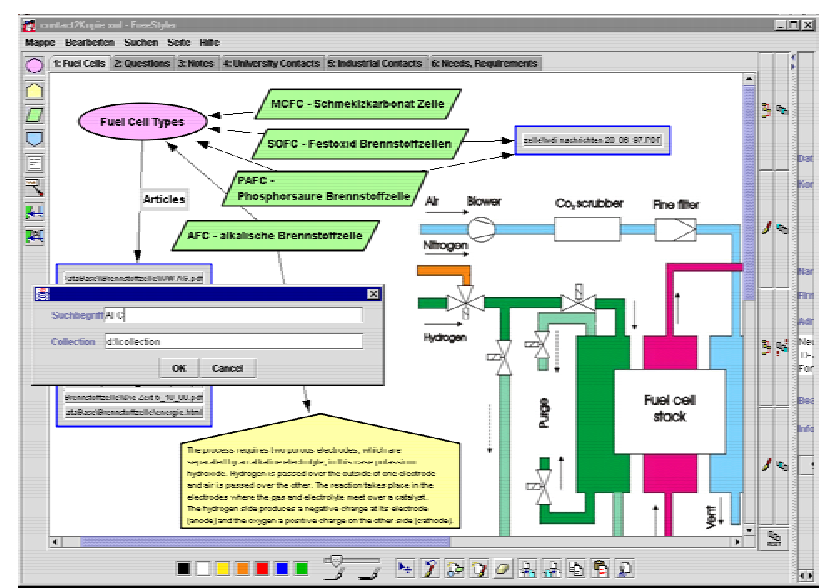

Figure 7

Figure 8 shows the possible use of FreeStyler for concept mapping enhanced with free hand annotations. The dialogue supports the request for files that are associated with special keywords. Based on the result, the concepts in this map can be related to available other material.

Figure 9 is an example of a comprehensive representation that is easily re-usable. Based on a geographical map, internal links are added which show where contacts to fuel cell producers are available. The links point to other FreeStyler pages that give more information about this concrete company.

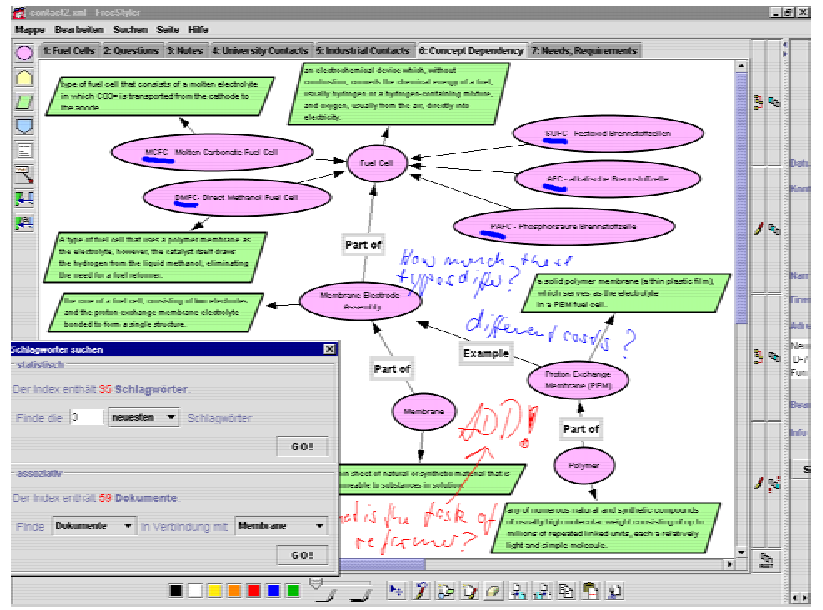

Figure 8

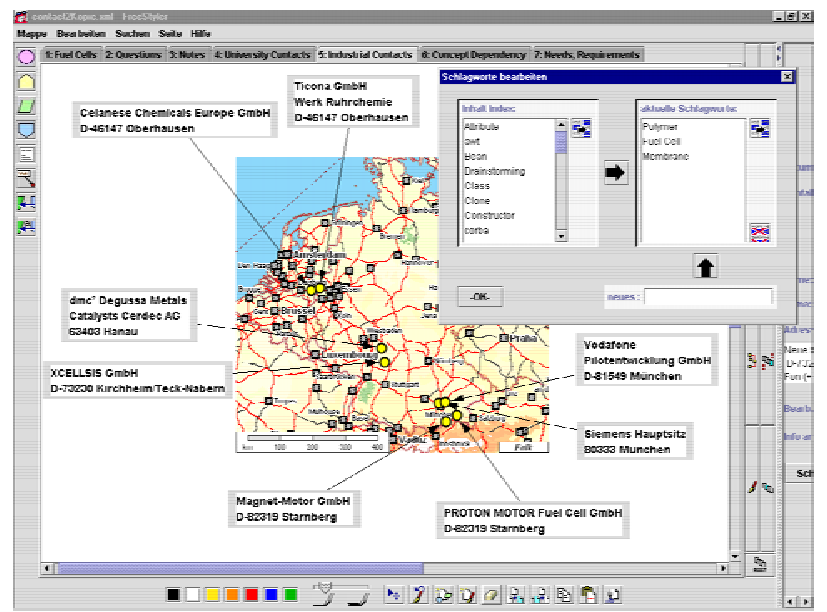

Figure 9

Through a dialogue, a FreeStyler map can be indexed with keywords. Already existing keywords are shown on the left. Putting a new keyword, the list on the left goes to alphabetically closest existing word to avoid the entry of similar and potentially redundant keywords (synonyms - homonyms). 


\section{DISCUSSION}

FreeStyler is currently being evaluated in everyday use in our TTD. At a first glance, organising technology transfer is still a tough problem, in contrast to which the use of FreeStyler is smooth and straightforward. Detailed evaluation results will be available soon.

We see the following analogies between our case study in trend monitoring and general functions for collaborative learning environments:

- The "trend monitoring" activity corresponds to learning activities in which students themselves explore a given theme or problem by searching relevant information, associating and elaborating this information and by documenting the results. This is typical for problem-based learning (Koschmann et al., 1996), but also for other types of open learning situations.

- "Trends" in our scenario correspond to "themes" in learning situations. FreeStyler allows for treating themes as moving targets, i.e., for moving from one are of interest to others, while maintaining a structured record of what has been done.

- The system is open (see requirements formulated above) in that it can search and integrate external sources in arbitrary format as long as the standard search engine is able to handle these. References to external sources are objects within the system

- The system maintains a base of index terms in the form of a rudimentary thesaurus. We would implement a full thesaurus support only if practically needed. Yet, we have already identified one very interesting feature: The detection of new terms in our TTD application may indicate a new trend (an "innovation") which is usually much more interesting than the assignment of an already existing terms. Accordingly, in learning, the appearance of a new theme may indicate new learning opportunities and may trigger certain types of "innovative" group processes.

Technically, the FreeStyler system is currently unified with the new version of the CardBoard environment which supports various palettes of languages at a time (e.g. to facilitate modelling). Also this opens new application perspectives.

\section{ACKNOWLEDGMENTS}

We thank Jörg Dixkens and his colleagues from the TTD for his valuable input and insightful suggestions. In the implementation work we were strongly supported by our student collaborators Martin Fendrich and Stefan Buschmann. This work was partially supported by grants from the Ministry of Science and Technology of the Federal State of Nordrhein-Westfalen and Mannesmann Pilotentwicklung (now Vodafone).

\section{REFERENCES}

Borghoff, U. M. \& Pareschi, R. (1998). Information technology for knowledge management. Berlin, Heidelberg, New York: Springer.

Conklin, J. \& Begemann, M. L. (1987). gIBIS: A hypertext tool for team design deliberation. In Proceedings of Hypertext'87 (pp. 247-251). Chapel Hill (North Carolina).

Constantino-Gonzalez, M. A., Suthers, D. (2000). A coached collaborative learning environment for EntityRelationship modeling. In Gauthier, G., Frasson, C., VanLehn, K. (eds.), Proceedings of the $5^{\text {th }}$ International Conference on Intelligent Tutoring Systems ITS 2000 (pp. 252-261), Montreal, June 2000: Springer.

Gaßner, K., Tewissen, F., Mühlenbrock, M., Loesch, A. \& Hoppe, H. U. (1998). Intelligently supported collaborative learning environments based on visual languages: a generic approach. In Darses, F. \& Zaraté, P. (eds.), Proceedings of $3^{\text {rd }}$ International Conference on the Design of Cooperative Systems (pp. 47-55). Cannes (Frankreich).

Gaßner, K. (2001). Architecture of a Cooperative Discussion Environment based on Visual Languages. In. Dillenbourg, P., Eurelings, A. \& Hakkarainen, K. (eds.). Proceedings of the European Conference on Computer-Supported Collaborative Learning (EuroCSCL-2001) (pp. 261-268). Maastricht, The Netherlands, March 2001.

Hoppe, H. U., Gaßner, K., Mühlenbrock, M. \& Tewissen, F. (2000). Distributed visual language environments for co-operation and learning: Applications and intelligent support. In Group Decision and Negotiation, 9(3), 205-220. 
v. Joolingen, W. (2000). Designing for collaborative discovery learning. In Gauthier, G. Frasson, C. \& VanLehn, K.(eds.), Proceedings of the $5^{\text {th }}$ International Conference on Intelligent Tutoring Systems ITS 2000 (pp. 202-211). Berlin et al.: Springer.

Koschmann, T., Kelson, A.C., Feltovich, P.J. \& Barrows, H.S. (1996). Computer-supported problem-based learning: a principled approach to the use of computers in collaborative learning. In Koschmann, T.D. (ed.). CSCL: Theory and Practice of an Emerging Paradigm (pp. 83-124). Hillsdale, NJ: Lawrence Erlbaum.

Lakin, F. (1990). Visual languages for co-operation: a performing medium approach to systems for cooperative work. In Galegher, J., Kraut, R. \& Egido, C. (eds.). Intellectual Teamwork (pp. 453-488). Hillsdale, NJ: Lawrence Erlbaum.

Nonaka, I. (1994). A Dynamic Theory of Organisational Knowledge Creation. Organization Science 5 (1), 14-37.

Pinkwart, N., Hoppe, H.U., \& Gaßner, K. (2001). Integration of domain-specific elements into visual language based collaborative environments. Accepted for Proceedings of CRIWG 2001 (Int.'l Workshop on Groupware, Darmstadt, Germany, September 2001).

Scardamalia, M. \& Bereiter, C. (1992). An Architecture for Collaborative Knowledge Building. In De Corte, E., Linn, M.C., Mandl, H. \& Verschaffel, L. (eds.), Computer-Based learning environments and problem solving (NATO ASI Series, Vol. 84) (S. 41-66). Berlin Heidelberg: Springer-Verlag.

Scardamalia, M., Bereiter, C., Brett, C., Burtis, P. J., Calhoun, C. \& Smith Lea, N. (1992). Educational applications of a networked communal database. Interactive Learning Environments, 2(1), 45-71.

Selvin, A., Buckingham Shum, S., Sierhuis, M., Conklin, J., Zimmermann, B., Palus, C., Drath, W., Horth, D., Domingue, J., Motta, E., Li, G. (2001). Compendium: Making Meetings into Knowledge Events. The Open University, Milton Keynes (UK), KMI-TR-103 (www.kmi.open.ac.uk/tr/papers/kmi-tr-103.pdf).

Stahl, G. (2000). Collaborative information environments to support knowledge construction by communites. AI \& Society 14, 1-27.

Streitz, N., Geißler, J., Haake, J. \& Hol, J. (1998). DOLPHIN: Integrated meeting support across Liveboards, local and remote desktop environments. In Fankhauser, P. \& Ockenfeld, M. (eds.), Integrated publication and information systems. (S. 57-74). Darmstadt: GMD IPSI.

Streitz, N., Haake, J., Hannemann, J., Lemke, A., Schuler, W., Schütt, H. \& Thüring, M. (1992). SEPIA: A cooperative hypermedia authoring environment. In Proceedings of the $4^{\text {th }} A C M$ Conference on Hypertext(ECHT'92) (pp. 11-22). Milano (Italy).

Suthers, D. D. (1999). Representational bias as guidance for learning interactions: A research agenda. In Lajoie, S. P. \& Vivet, M. (eds.), Artificial intelligence in education (pp. 121-128). Amsterdam: IOS Press.

Suthers, D., Toth, E. E., and Weiner, A. (1997). An integrated approach to implementing collaborative inquiry in the classroom. In Proceedings of CSCL '97 (Int.'1 Conf. on Computer Supported Collaborative Learning, Toronto, Canada, December 1997), 272-279.

Suthers, D., Weiner, A., Connelly, J. \& Paolucci, M. (1995). Belvedere: Engaging students in critical discussion of science and public policy issues. In Greer, J. (ed.), Proceedings of the 7th World Conference on Artificial Intelligence in Education_(pp. 266-273). Whashington DC (USA).

TrendMonitoring (1999). Funded by the Ministry for Schools, Science, Economy and Research of the State Nordrhein-Westphalen (Gemany). http://www.uni-duisburg.de/THP/trendmonitoring/

Wagler, F. (1998). Erstellung eines Petrinetz-basierten Steuerungsmechanismus zur Behandlung von Turn-TakingRegeln in Gruppen-interaktiven Spielen (Development of a petri net based controlling mechanism for the handling of turn-taking rules in interactive group games). Unpublished masters thesis. University of Duisburg, Department of Mathematics and Computer Science. 